\title{
O PLANEJAMENTO ESTRATÉGICO NO SETOR PÚBLICO BRASILEIRO ${ }^{1}$
}

\author{
STRATEGIC PLANNING IN THE BRAZILIAN PUBLIC SECTOR
}

\section{PLANIFICACIÓN ESTRATÉGICA EN EL SECTOR PÚBLICO BRASILEÑO}

Luiza Szczerbacki Castello Branco, Advogada formada pela UFF (Universidade Federal Fluminense) e Mestre em Administração Pública pela Fundação Getulio Vargas. Endereço Profissional: Rua Pereira da Silva, n.586/801, Laranjeiras, Rio de Janeiro, RJ - Brasil. CEP 22221-140. Telefone: 21 99978-1988, 21 2265-1818. Email: luizabranco@ig.com.br.

\section{RESUMO}

O presente ensaio tem como objetivo incentivar a implantação do planejamento estratégico no setor público brasileiro. O escopo das reformas da década de 1980, por meio do movimento denominado New Public Management (NPM), era transpor as técnicas gerenciais do setor privado para o público. Dentre esses métodos, dar-se-á ênfase ao planejamento estratégico na seara estatal, que ainda é pouco explorado pela ciência administrativa nacional. Escassos estudos no Brasil focam na investigação dos resultados deste processo nas organizações governamentais ou desenvolvem teorias sobre o tema. Considerando essa lacuna teórica, o presente ensaio objetiva discutir possíveis diferenças entre os setores público e privado de forma a caracterizar o planejamento estratégico governamental brasileiro como necessariamente distinto do particular. Com base na teoria da estratégia, o estudo explora as possibilidades da teoria do planejamento estratégico, utilizando a metodologia bibliográfica, descritiva e explicativa. Por fim, os resultados apontam que esse planejamento quando corretamente adaptado às organizações públicas nacionais é benéfico para $\mathrm{o}$ seu desenvolvimento.

Palavras-Chave: Estratégia; Planejamento Estratégico; Setor Público.

\begin{abstract}
This essay aims to encourage the implementation of strategic planning in the Brazilian public sector. The scope of the reforms of the 80's, through the movement called New Public Management (NPM), was transport management techniques from the private to the public sector. Among these methods, it will emphasize strategic planning state, which it still trifling by the national science management. Few research studies in Brazil focus on the results of implementing this process in governmental institutions or develop theories on the subject. Considering this theoretical gap, this paper discusses possible differences between the public and private sectors in order to characterize the Brazilian public strategic planning as necessarily distinct from the private. Based on the theory of strategy, this study explores the possibilities of the theory of strategic planning, using the method of literature research, by descriptive and explicative methodology. Finally, the results indicate that this planning when properly adapted to the national public's organizations, is beneficial to their development.
\end{abstract}

\footnotetext{
${ }^{1}$ Artigo submetido em 28/01/2014, revisado em 04/02/2014, aceito em 20/02/2014 e divulgado em 01/05/2014 pelo Editor João Carlos Hipólito Bernardes do Nascimento, após double blind review.
}

GєCont, v. 1, n. 1, Floriano-PI, Jan-Jun. 2014. 
Keywords: Public Sector; Strategic Planning; Strategy.

\section{RESUMEN}

Este artículo tiene como objetivo fomentar la aplicación de la planificación estratégica en el sector público brasileño. El objetivo de las reformas de la década de 1980, a través del movimiento llamado "New Public Management (NPM)" - la Nueva Gestión Pública (NGP), fue cruzar las técnicas de gestión del sector privado al público. Entre estos métodos, se le dará énfasis a la planificación estratégica del estado, que todavía está inexplorado por la ciencia administrativa nacional. Pocos estudios en Brazil se centran en la investigación de los resultados de este procedimiento en las organizaciones gubernamentales brasileñas o desarrollan teorías sobre el tema. Dado este vacío teórico, este trabajo busca discutir las posibles diferencias entre los sectores público y privado con el fin de caracterizar la planificación estratégica brasileña del gobierno necesariamente distinta de lo particular. Sobre la base de la teoría de la estrategia, el estudio explora las posibilidades de la teoría de la planificación estratégica, utilizando la metodología bibliográfica, descriptiva y explicativa. Por último, los resultados indican que esta planificación cuando se adaptó adecuadamente a las organizaciones públicas nacionales, es beneficiosa para su desarrollo.

Palabras clave: Estrategia; Planificación Estratégica; Sector Público.

\section{INTRODUÇÃO}

$\mathbf{O}$ termo estratégia requer um conjunto de definições para reproduzir com clareza a sua amplitude (MINTZBERG, 1987), eis que tem sua origem datada de aproximadamente 500 a.C. e, desde então tem evoluído, passando por transformações e adaptações (MOTTA, 2012).

De origem grega, o estratego era o comandante militar que formulava e projetava as manobras necessárias à consecução de um fim específico. Com o passar dos tempos, contudo, a ciência da administração transpassou o conceito de estratégia sob o ângulo militar para uma perspectiva gerencial empresarial moderna (DARÓS, 2009).

O conceito de estratégia começou a ser usado em administração nos anos 1950, com o intuito de incutir nas empresas privadas uma nova perspectiva de futuro e já na década de 1960, havia se popularizado no meio empresarial associado ao planejamento (MOTTA, 2012). Na década de 1970, a introdução e utilização do planejamento estratégico nas organizações não subordinadas ao Estado havia claramente se acelerado e se difundido (BAZZAZ; GRINYER, 1981).

Assim, enquanto o setor privado evoluía com as novas técnicas e processos, o setor público mantinha-se estagnado, arraigado às práticas obsoletas e burocráticas. Houve então, um movimento amplo de reformas na gestão pública, intensificado no mundo a partir dos anos 1980 e denominado New Public Management - NPM, cujo objetivo fundamental era levar técnicas de gestão da seara privada para a pública (CAMPOS, 1990; OSBORNE; GAEBLER, 1996; SHICK, 1996; DROR, 1999; POLLIT; BOUCKAERT, 2004; ABRUCIO, 2005; CAVALCANTI, 2005; ABRUCIO; SANO, 2008; PECI; PIERANTE; RODRIGUES, 2008; COSTIN, 2010; CUNHA, 2010).

Considerando o breve cenário apresentado, o presente ensaio teórico procura incentivar a implantação do planejamento estratégico, processo que foi desenvolvido pelas

GєCont, v. 1, n. 1, Floriano-PI, Jan-Jun. 2013. 
empresas privadas, no setor público brasileiro. O objetivo é propor a reflexão sobre a transposição deste método de um setor para o outro, tema que tem sido alvo de diminuta atenção dos pesquisadores pátrios.

Ademais, busca-se apontar peculiaridades e diferenças entre as referidas áreas sob o ponto de vista gerencial, levantando-se questões para serem estudadas em futuras pesquisas. A seguir são apresentadas as bases teóricas da estratégia e do planejamento estratégico, juntamente com uma breve descrição teórica do setor público e privado. Em seguida, as análises são sucintamente discutidas, sendo concluídas nas considerações finais.

\section{FUNDAMENTAÇÃO TEÓRICA}

\subsection{Estratégia}

O conceito de estratégia é amplo e dinâmico, ou seja, se altera dependendo da situação, do contexto e da cultura em que as organizações ou pessoas se encontram (QUINN, 2006) (para diferentes pontos de vista, ver, por exemplo, MINTZBERG, 1987; SIMONS, 1994; CERTO; PETER, 1993; ANDREWS, 2001). Todas essas diversas abordagens, no entanto, têm por finalidade garantir que a organização alcance os objetivos pretendidos.

O conceito de Porter (1986), segundo o qual a estratégia é uma combinação dos fins as metas que a organização busca - e dos meios - as políticas pelas quais a referida empresa está buscando chegar lá -, sintetiza a conotação que este trabalho atribuirá a este termo. No mais, cumpre destacar que "estratégia não é consequência do planejamento, mas o oposto: ela é o ponto de partida" (MINTZBERG, 1994a, p.333). Formalizando essa perspectiva, tem-se o planejamento estratégico, que vê a formulação da estratégia como um processo de planejamento formal separado e sistemático (BRAGA, 2003).

\subsection{Planejamento Estratégico}

O planejamento sempre foi uma das funções básicas da administração. Tanto assim que um dos quatro princípios da administração científica de Taylor era o planejamento (os demais eram: seleção ou preparo, controle e execução). Nesse sentido, o francês Henry Fayol estabeleceu como primeira função do gestor a previsão, ou seja, visualizar o futuro desenvolvendo um plano de ações para atingir as metas traçadas (FLEURY, 2005).

O setor privado, necessitando acompanhar as rápidas alterações trazidas pela modernização da sociedade e pela concorrência cada vez mais acentuada dos mercados, a partir da metade dos anos 1960, introduziu em suas organizações os conceitos básicos da metodologia do planejamento estratégico, mediante as proposições do professor Igor Ansoff (TAYLOR, 1975; MINTZBERG, 1994B).

Segundo o próprio Ansoff (1969), o planejamento estratégico consiste em uma série de diretrizes administrativas que especificam a posição da organização no mercado, as direções nas quais ela procura desenvolver-se e os instrumentos competitivos que ela empregará. É um processo contínuo de aprendizado, sistematicamente e com maior conhecimento possível do futuro capaz de medir o resultado de decisões de risco em confronto com as expectativas alimentadas (DRUCKER, 1984).

É através do exercício de planejar que se aprende sobre as demandas e necessidades externas e sobre a capacidade de resposta da empresa. Mesmo quando não implementados, os planos revelam as expectativas e referências de valor, essenciais a um grupo de trabalho (MOTTA, 2012).

GєCont, v. 1, n. 1, Floriano-PI, Jan-Jun. 2013. 
O planejamento estratégico tem por objetivo, portanto, posicionar de forma competitiva a organização em seu ambiente, fornecendo a direção que deve ser seguida, sendo crucial para o sucesso em longo prazo de toda instituição (MARCOVITCH; VASCONCELOS, 1977; MARCOVITCH; RADOSEVICH, 1978).

No início da década de 1970, o conceito de gerência estratégica foi introduzido para fazer face aos desafios de uma nova época de mudanças extremamente velozes e de grande magnitude. A prática do planejamento estratégico, principalmente no que concerne às suas propostas racionais de análise antecipatória, parecia ineficaz para responder às demandas do novo momento.

Por outro lado, passou-se a enfatizar a importância do planejamento estratégico nas suas perspectivas de contínuo, sistemático e contingencial, aceitando-o mais como um processo emergente de um conjunto de decisões e ações do que como algo antecedente ao processo de implementação. Para caracterizar essa mudança, surge assim o conceito de gerência estratégica (MOTTA, 2012).

Sendo assim, as estratégias devem resultar de um processo controlado e consciente de planejamento formal, decomposto em etapas distintas, cada uma delineada por checklists e apoiada por técnicas específicas. Surgem prontas deste processo, devendo ser explicitadas para que possam ser implantados através da atenção detalhada a objetivos e metas, orçamentos, programas e planos operacionais de vários tipos (MINTZBERG; AHLSTRAND; LAMPEL, 2000).

A responsabilidade por todo este processo está, em princípio, com o executivo principal, sendo deliberado pelos níveis hierárquicos mais altos da instituição (MARCOVITCH; VASCONCELOS, 1977; MINTZBERG; AHLSTRAND; LAMPEL, 2000). Na prática, contudo, a responsabilidade pela execução é dos planejadores ou gestores, nos níveis hierárquicos mais baixos (BAZZAZ; GRINYER, 1981).

\subsection{Planejamento Estratégico no Setor Público}

Diversas foram às organizações privadas que adotaram o processo de planejamento no mundo inteiro. Muitas delas, porque bem feita a sua formulação, execução e controle, alcançaram resultados positivos (HEROLD, 1972; MARCOVITCH; RADOSEVICH, 1978; MARIN, 2012).

Da mesma forma que no setor privado, devido às frequentes e rápidas mudanças no ambiente, além do contexto de transformações radicais da sociedade e do Estado, impôs-se, também, a modernização do setor público (DARÓS, 2009). Diversos autores (OSBORNE; GAEBLER, 1996; PETERS; PIERRE, 1998; MILWARD; PROVAN, 2000) enfatizaram a necessidade de mudanças nos governos, tendo em vista as transformações que vêm se perpetrando nas últimas décadas enquanto os Estados continuam estáticos.

Destarte, a partir da década de 1990, no contexto das reformas dos órgãos do Estado brasileiro, sob o foco da administração gerencial, passou a ser relevante a capacidade de gerenciamento (CAVALCANTI, 2005). A gestão na atividade pública tornou-se tão importante quanto no setor privado (MARIN, 2012), caracterizando-se por uma despolitização da administração, aliada à ênfase no conceito de eficiência governamental (FLEURY, 2005).

Osborne e Gaebler (1996), importantes autores do corpo de ideias conhecido como New Public Management (NPM), a partir da década de 1980 influenciaram e incentivaram reformas administrativas em diversos governos nacionais. Para os autores, o governo poderia se apropriar do modelo de fornecimento de serviços do setor privado, como, por exemplo, a 
flexibilidade de gestão, o foco na qualidade dos serviços e a prioridade às demandas dos consumidores.

Desta forma, na gestão pública, a introdução do planejamento estratégico faz referência ao movimento da New Public Management (SHICK, 1996), validando-o como referência para a tomada de decisões sobre como estruturar e gerir os serviços públicos (BARZELAY, 2001).

A administração gerencial tem suas premissas voltadas para a eficiência, agilidade, qualidade e flexibilidade da gestão além da preocupação com o aumento da responsabilização da administração pública (CAMPOS, 1990; DROR, 1999; POLLIT; BOUCKAERT, 2004; ABRUCIO, 2005; CAVALCANTI, 2005; ABRUCIO; SANO, 2008; CUNHA, 2010), fortalecimento do gerenciamento estatal e imputação de resultados (COSTIN, 2010). Tais conceitos e práticas passariam a nortear o modelo gerencial público, direcionando a adaptação e a transferência dos conhecimentos desenvolvidos nas empresas para a seara governamental (PECI, PIERANTE; RODRIGUES, 2008).

Em síntese, prevalecia a ideia de maior eficiência das organizações privadas que necessitavam se desenvolver intensamente para sobreviver no ambiente competitivo. Considerando que em toda gestão, privada ou pública, existem desafios semelhantes, as técnicas e conceitos que auxiliaram as empresas a se tornarem ágeis e eficientes poderiam, consequentemente, ser utilizadas também no setor público (FREDERICKSON, 1989; OSBORNE; GAEBLER, 1996).

Todavia, a $N P M$ recebeu muitas críticas, dentre as quais podem-se destacar a pretensão de se constituir numa prática universal, aplicável às diversas realidades, independentemente, das especificidades de cada local; a supressão de valores como a cidadania, equidade e bem-estar público ao deslocar a atenção para conceitos mais restritos como o de eficiência e produtividade; e a desconsideração das diferenças essenciais existentes entre a administração pública e privada (DIEFENBACH, 2009).

Apesar das teorias e conceitos de planejamento fornecerem algumas diretrizes gerais, as circunstâncias de cada organização (tanto as públicas quanto as privadas) são tão específicas que a administração precisa adaptar estas generalizações quando da aplicação do planejamento estratégico. A adoção de um processo prescritivo, sem a devida adequação para a realidade organizacional de cada tipo de instituição, pode corresponder a um desperdício de tempo e recursos (MARCOVITCH; RADOSEVICH, 1978).

\subsection{Setor Público X Setor Privado}

Como visto, existem dois setores bem distintos em um Estado, o setor público, voltado para o interesse da sociedade, sem intuito de lucratividade e com estabilidade empregatícia, e o setor privado, de interesse do empresariado, visando o lucro e com alta rotatividade dos trabalhadores no mercado (MARCONI, 1997; UCHITELLE, BATTENBERG III; KOCHAN, 2007) (para mais estudos com diferenças entre tais setores, ver, por exemplo, PAGANI, 2003; ALLISON, 2002; APPLEBY, 2002; DUNLOP, 1979; RIANEY, BACKOFF; LEVINE, 1976; NEUSTADT, 1979; MARKOVITS, DAVIS; FAY; DICK, 2010).

Ademais, o alto grau de formalismo, legalismo e rigidez das práticas rotineiras das organizações públicas acarretam uma dinâmica mais lenta em relação às organizações privadas, pois o seu negócio é prover ou contratar por meio de serviços, em geral não lucrativos, funções obrigatórias que têm para com a população ou governo (MARIN, 2012).

Além disso, as organizações governamentais estão inseridas num sistema social complexo e conflitivo (DARÓS, 2009), onde a submissão de decisões oriundas de setores 
eminentemente técnicos ao crivo do interesse político do governo impede, em alguns casos, o desenvolvimento pleno das atividades dos seus funcionários (BELLANTE; LINK, 1981; MARIN, 2012).

No mais, as empresas públicas também são conhecidas por sua ineficiência, resultado de pressões políticas dos governantes que as controlam e pela desídia de seus trabalhadores que dificilmente são demitidos (SHLIFER; VISHNY, 1994).

\section{METODOLOGIA DA PESQUISA}

A metodologia utilizada neste ensaio foi, quanto aos fins, descritiva, ao expor características de determinado fenômeno, estabelecendo correlações e definindo sua natureza; e explicativa, na medida em que explica os eventos descritos. Quanto aos meios, a pesquisa foi tão somente bibliográfica (VERGARA, 2011).

\section{APRESENTAÇÃO E DISCUSSÃO DOS RESULTADOS}

Partindo da análise de que o setor público nacional está em busca de eficiência e que as relações e decisões deste setor são em sua maioria de cunho político, patrimonialistas e burocráticas (BRESSER-PEREIRA, 2000), a inserção do planejamento estratégico nestas organizações pode ser percebido de forma positiva.

A estratégia, instrumento essencial para o desenvolvimento das empresas privadas, por meio de planejamentos estratégicos, conseguiu aumentar a sua produtividade, eficiência e lidar com a volatilidade dos mercados privados.

O setor público brasileiro por seu turno manteve-se fragmentado pelos diferentes governos que assumiam o poder, sem continuidade das políticas públicas que eram adotadas, nem objetivos traçados em longo prazo. Corroborando para a mantença dessa situação, a estabilidade empregatícia fazia com que os servidores não fossem tão comprometidos com o trabalho, pois o risco de demissão era ínfimo.

Deste modo, a inclusão do planejamento estratégico no setor público nacional pode trazer a integração, em todos os níveis das organizações estatais, do contínuo processo de formulação, implementação e avaliação do futuro da instituição com um horizonte de tempo razoável para a obtenção dos resultados almejados. Com a implantação de metas a serem cumpridas, tira-se da inércia os funcionários que passam a ter que enfrentar situações com as quais não estavam acostumados. Daí surge a necessidade de importar as técnicas e processos do setor privado.

Finalmente, cabe refletir se essa transmissão, que vem se perpetrando desde o final da década de 1990 no Brasil, das práticas adotadas nas empresas privadas para as instituições públicas está sendo feita corretamente. Não se deve olvidar que ambas as áreas são distintas, têm escopos diferentes e peculiaridades próprias, devendo tais fatores serem levados em consideração quando da formulação dos planejamentos públicos. Ademais, deve-se refletir se não seria melhor se falar em um planejamento estratégico propriamente público, como um processo autônomo do planejamento privado, haja vista que atualmente possuem mais diferenças do que similitudes. 


\section{CONSIDERAÇÕES FINAIS}

O presente trabalho objetiva contribuir para o estudo do planejamento estratégico no setor público nacional por meio de uma abordagem reflexiva. Emergindo do referencial da teoria da estratégia e do planejamento estratégico, o estudo apresenta uma reflexão de como a estratégia contemporânea, por meio do planejamento, valoriza a eficiência e o impacto da organização no aproveitamento dos recursos disponíveis e na satisfação de demandas e de necessidades, principalmente as comunitárias.

Os governos devem otimizar os recursos disponíveis de forma a obter cada vez mais e melhores resultados a menores custos. Para tanto, o caminho mais adequado mostra-se o de elaboração de planejamentos estratégicos.

Buscou-se, também, avaliar as diferenças entre as searas pública e privada, devido às diversas especificidades entre elas. A partir desta ponderação, é possível analisar como o planejamento estratégico, não obstante buscar a eficiência das organizações, deve ser diverso nesses dois setores.

Por fim, concluiu-se que o planejamento no setor público é necessário para o bom funcionamento do Estado moderno no Brasil, sendo benéfico quando devidamente adaptado à instituição, e inócuo, na medida em que não é corretamente amoldado às peculiaridades das organizações estatais nacionais.

Reconhecendo as limitações do presente trabalho, tanto em relação ao tipo de estudo quanto à abordagem teórica, levanta-se um assunto atual e recorrentemente percebido pela administração pública, porém pouco desbravado na teoria nacional. Sendo assim, propõe-se que exista um planejamento estratégico próprio do setor público brasileiro, distinto do privado, contudo, este ainda resta carecedor de teorias e estudos mais aprofundados. Futuras pesquisas poderiam, portanto, abordar as singularidades do setor público brasileiro assim como esmiuçar teorias acerca do planejamento estratégico na seara governamental além de propor formas de melhor adequá-lo às instituições nacionais.

\section{REFERÊNCIAS}

ABRUCIO, F. L.. Reforma do Estado no federalismo brasileiro: a situação das administrações públicas estaduais. Revista de Administração Pública, v. 39, n. 2, p. 401420, 2005.

; Sano, H.. Promessas e Resultados da Nova Gestão Pública no Brasil: O caso das Organizações Sociais de Saúde em São Paulo. Revista de Administração de Empresas, v. 48, n. 3, p. 64-80, 2008.

ALLISON, G.. Public and Private Management: Are they fundamentally alike in all unimportant respects? In: J. M. Shafritz; A. C. Hyde; S. J. Parkes. Classics of Public Administration, 5th Edition, Thomson-Wadsworth, 2002.

ANDREWS, K. R.. O conceito de estratégia empresarial, In: H. Mintzberg; J. B. Quinn. O processo da estratégia. 3 ed. Porto Alegre: Bookman, 2001.

ANSOFF, I.. Business Strategy. England, Penguin Books, 1969.

APPLEB, P.. Government is different. In. J. M. Shafritz; A. C. Hyde; S. J. Parkes. Classics of Public Administration, 5th ed., Thomson-Wadsworth, 2002.

GєCont, v. 1, n. 1, Floriano-PI, Jan-Jun. 2013. 
BARZELAY, M.. The New Public Management. Improving Research and Policy Dialogue. University of California Press/Russel Sage Foundation, 2001.

BAZZAZ, S.; GRINYER, P.. Corporate Planning in the U.K.: The State of the Art in the 70s. Strategic Management Journal, v. 2, n. 2, 1981.

BELLANTE, D.; LINK, A. N.. Are public sector workers more risk averse than private sector worker? Industrial \& Labor Relations Review, v. 34, n. 3, p. 408-412, 1981.

BRAGA, R. T. Planejamento Estratégico e o Controle da Gestão Pública: Desenvolvimento da ação de Controle da Gestão dos Recursos Públicos. 125f. Dissertação (Mestrado em Administração) - Escola Brasileira de Administração Pública e de Empresas, Fundação Getulio Vargas, Rio de Janeiro, 2003.

BRESSER-PEREIRA, L. C.. A Reforma Gerencial do Estado de 1995. Revista de Administração Pública, v. 34, n. 4, p. 55-72, 2000.

CAMPOS, A. M.. Accountability: Quando poderemos traduzi-la para o Português? Revista de Administração Pública, v. 24, n. 2, p. 30-50, 1990.

CAVALCANTI, B. S.. O Gerente Equalizador. Estratégias de Gestão no Setor Público. Rio de Janeiro: Editora FGV, 2005.

CERTO, S.; PETER, J. P.. Administração estratégica: Planejamento e implantação da estratégia. São Paulo: Makron Books, 1993.

COSTIN, C.. Administração Pública. Rio de Janeiro: Elsevier, 2010.

CUNHA, A.. A Busca de Maior Vitalidade da Gestão nas Organizações do Poder Judiciário. Cadernos FGV Projetos, ano 5, n. 12, p. 45-51, 2010.

DARÓS, V.. Planejamento Estratégico do TRF da 4 Região. Caderno de Administração da Justiça: Planejamento Estratégico, 64p, 2009.

DIEFENBACH, T.. New public management in public sector organizations: the dark sides of managerialistic 'enlightenment'. Public Administration, v. 87, p. 892-909, 2009.

DROR, Y.. A Capacidade para Governar. Informe ao Clube de Roma. Edições FUNDAP, 1999.

DRUCKER, P.. Introdução à administração. São Paulo: Futura, 1984.

DUNLOP, J. T.. Public Management. Unpublished paper. In: J. M. Shafritz; A. C. Hyde; S. J. Parkes. Classics of Public Administration, 5th ed., Thomson-Wadsworth, 1979.

FLEURY, N. A qualificação do modelo "linha de frente-retaguarda" e sua adaptabilidade às características da administração judiciária. In: A. Cunha; P. Motta. A reforma do Poder Judiciário no estado do Rio de Janeiro/Fundação Getulio Vargas Rio de Janeiro: Fundação Getulio Vargas, p. 83-92, 2005.

FREDERICKSON, H. G.. Changing Epochs of Public Administration. Public Administration Review, v. 49, n. 2, (special issue), 1989.

HEROLD, D.. Long-Range Planning and Organizational Performance: A CrossValuation Study. Academy of Management Journal, v. 15, n. 1, 1972.

MARCONI, N.. Uma breve comparação entre os mercados de trabalho do setor público e privado. Revista do Setor Público, v. 48, n. 1, 1997. 
MARCOVITCH, J.; VASCONCELLOS, E.. Técnicas de Planejamento Estratégico para Instituições de Pesquisa e Desenvolvimento. Revista de Administração, v. 12, n. 1, p.61-78, 1977.

; RADOSEVICH, R.. Planejamento Estratégico nas Organizações Estruturadas por Projeto. Revista de Administração, São Paulo, v. 13, n. 2, p. 24-39, 1978. Boston, v. 72, n. 1, p. 103-108, 1994 b.

Prentice-Hall, 1994a

. The Rise and Fall of Strategic Planning. Englewood Cliffs, NJ:

; AHLSTRAND, B.; LAMPEL, J.. Safári de estratégia: um roteiro pela selva do planejamento estratégico. Trad. Nivaldo Montingelli Jr., Porto Alegre: Bookman, 2000 .

; QUINN, J. B.. O processo da estratégia. Trad. James Sunderland Cook, 3 ed., Porto Alegre: Bookman, 2001.

MARIN, J.C.. The impact of strategic planning and the balanced scorecard methodology on middle managers' performance in the public sector. International Journal of Business \& Social Science, v. 3, n. 1, 2012.

MARKOVITS, Y.; DAVIS, A. J.; FAY, D.; DICK, R. . The Link Between Job Satisfaction and Organizational Commitment: Differences Between Public and Private Sector Employees. International Public Management Journal, v. 13, n. 2, p. 177-196, 2010.

MILWARD, H. B.; PROVAN, K. G.. Governing the Hollow State. Journal of Public Administration Research and Theory, p. 193-314, 2000.

MINTZBERG, H.. The strategy concept: five ps for strategy. California Management Review, v. 30, n. 1, p. 11-24, 1987.

MOTTA, P. R.. Planejamento das Organizações Públicas, Apostila do Mestrado Acadêmico em Administração Pública, Rio de Janeiro, 2012.

NEUSTADT, R. E.. American Presidents and Corporate Executives. Panel on presidential management of the National Academy of Public Administration, Washington, D. C, 1979.

OSBORNE, D.; GAEBLER, T.. Reinventing Government: Introduction. In: J. M. Shafritz \& J. S. Ott. Classics of Organization Theory, Harcourt Brace, 1996.

PAGANI, L.. Why Do People from Southern Italy Seek Jobs in the Public Sector? LABOUR: Review of Labour Economics \& Industrial Relations, v. 17, n. 1, p. 63-91, 2003.

PECI, A.; PIERANTI, O. P.; RODRIGUES, S.. Governança e New Public Management: Convergências e Contradições no Contexto Brasileiro. Revista Organizações \& Sociedade, v. 15, n. 46, p. 39-55, 2008.

PETERS, B. G.; PIERRE, J.. Governance without government: rethinking public administration. Journal of Public Administration Research and Theory, v. 8, p. 223-244, 1998.

POllitT, C.; BOUCKAERT, G.. Public Management Reform. Oxford University Press, 2a Ed, 2004. 
PORTER, M. E.. Estratégia competitiva: técnicas para análise de indústrias e da concorrência. Trad. Elizabeth Maria de Pinho Braga, 7 ed., Rio de Janeiro: Campus, 1986.

QUINN, J. B.. Estratégias para a mudança. In: Mintzberg, H.; Lampel, J.; Quinn, J.B.; Ghoshal, S. O processo da estratégia: conceitos, contextos e casos selecionados. Porto Alegre, Ed. Bookman, 2006.

RAINEY, H. G.; BACKOFF, R. W.; LEVINE, C. H.. Comparing Public and Private Organizations. Public Administration Review, v. 36, n. 2, p. 233-244, 1976.

SHICK, A.. The Spirit of Reform: Managing the New Zealand State Sector in a Time of Change. Wellington, New Zealand: State Services Comission and the Treasury. Disponível em: www.sse.govt.nz. Acesso: 08 maio.2013, 1996.

SHLEIFER, A.; VISHNY, R. W.. Politicians and Firms. Quarterly Journal of Economics, v. 109, n. 4, p. 995-1025, 1994.

SIMONS, R.. Levers of control. Boston: Havard Business School Press, 1994.

TAYLOR, B.. Strategies for planning. Long Range Planning, Elmsford, p. 27-40, 1975.

UCHITELLE, L., BATTENBERG III, J. T., KOCHAN, T.. Employer-Employee Social Contracts: Fashioning a New Compact for Workers. Academy of Management Perspectives, v. 21, n. 2, p. 5-16, 2007.

VERGARA, S. C.. Projetos e relatórios de pesquisa em administração. 13 ed. São Paulo: Atlas, 2011. 